\title{
Do Americans Believe Modern Earth Science?
}

\begin{abstract}
Allan Mazur
Published online: 3 June 2010

(C) Springer Science+Business Media, LLC 2010
\end{abstract}

\section{Methods}

In 2006, the GSS asked a probability sample of over 1,600 adult respondents from the 48 contiguous United States if it was true or false that (1) Human beings, as we know them today, developed from earlier species of animals; (2) The universe began with a huge explosion; and (3) The continents on which we live have been moving their locations for millions of years and will continue to move in the future. (I take the "huge explosion" question as indicating whether or not the respondent accepts the big bang theory of the universe's origin, though the wording might be interpreted differently.) An additional question, which may serve as a baseline of misinformation, was (4) "Does the Earth go around the Sun, or does the Sun go around the Earth?"

Few of these respondents were scientists, and we cannot expect many to be able to cite with any precision the main lines of evidence that make evolution, the Big Bang, moving continents, and the sun-centered universe scientifically unassailable ideas. Respondents stated their beliefs as laypeople, telling if they accept these scientific claims as true or not.

There is a relativistic tradition in sociology to study conflicts in an even-handed way, treating each side's claims as legitimate from its own perspective. Analyzing World War II, for example, would require an understanding - an attempt to "get into the heads" - of Nazi and Japanese viewpoints as well as the Allied perspective. At least initially, the analyst does not treat either side as privileged or presume one stakeholder to be correct or singularly truthful.

This relativist tradition saves an analysis from becoming a polemic. It often demonstrates that the black-and-white perspective of a partisan has unrecognized grays and that

\footnotetext{
A. Mazur $(\bowtie)$

The Maxwell School, Syracuse University,

Syracuse, NY 13244, USA

e-mail: amazur@syr.edu
} 
the opposing sides see the world in very different ways. Often, it raises new doubts about which side is innocent and which blameworthy, or whose claims are truthful.

Such even-handed analysis becomes problematic as the weight of evidence favoring one side becomes overwhelming. While historians looking back at the sixteenth century would give equal voice to proponents of a geocentric universe versus a heliocentric one, few would remain open to a claim made today that the earth is the center of the solar system. Controversies in science and technology are often settled to nearly every informed person's satisfaction. In such cases, where - by reasonable criteria —one side is almost certainly right and the other wrong, relativism loses much of its value. Here, I presume that the above four scientific propositions are true and that respondents who believe otherwise are incorrect. My statistical analysis does not require this absolutist perspective, but with it the exposition becomes simpler.

The statistical analysis will show how beliefs about scientific ideas are related to three independent variables: education, religiosity, and political conservatism. Education is measured as the highest degree attained in school. Political conservatism is measured by response to the question,

I'm going to show you a seven-point scale on which the political views that people might hold are arranged from extremely liberal to extremely conservative. Where would you place yourself on this scale?

Responses of very or moderately liberal are coded one; responses of slightly liberal, middle of the road and slightly conservative are coded two; and responses of moderately or very conservative are coded three.

Social scientists have found the most useful classification of the USA's many religious denominations to be along a continuum from fundamentalism to liberalism. It is hard to place a rigorous definition on the poles of the continuum, but GSS administrators, using several criteria, provide a useful sorting of denominations into a three-category variable here called religiosity. Generally speaking, a fundamentalist denomination teaches the inerrancy of the literal Bible, word for word, because it is divinely written or inspired. A liberal denomination regards the Bible as some combination of myth, metaphor, and moral guidance, authored by humans, to be interpreted in accord with modern science and sensibilities. "Moderate" is a middle category, applied to denominations not clearly at either extreme. For details on the categorization of Protestant denominations, see Smith (1987). Using similar criteria, the
GSS puts Catholicism among moderate denominations because it teaches that the Bible cannot be understood literally, but is subject to interpretation by the Church. The GSS places Judaism among liberal denominations, reflecting the non-literal view of the Bible held by most American Jews. Of course, individuals within any one denomination vary in their beliefs, but the GSS sorting has proven its worth and is well-accepted.

Ordinary least-squares (OLS) regression analysis is a statistical technique that assesses how a dependent variable (e.g., belief versus disbelief that humans evolved from earlier species) is related to one or more independent variables (here religiosity, education, and political conservatism). The analysis produces a coefficient for each independent variable, telling how strongly it is related to the dependent variable (independently of the other independent variables). The analysis also produces a significance level for the relationship of each independent variable to the dependent variable.

Since the independent variables have different ranges, it is useful to express their coefficients in standardized form, called "betas." By comparing betas for the different independent variables, one can see which is most (and least) strongly related to the dependent variable. A large beta implies that the independent variables strongly predict - are strongly related to - the dependent variable.

For technical reasons, when the dependent variable is binary (e.g., true/false), OLS regression somewhat distorts significance levels. An alternate technique, logit analysis, is more accurate but also more difficult to interpret (Agresti 2002). For ease of interpretation, I show results from OLS regressions. Logit analyses, done separately but not shown, give similar significance levels, so there is no distortion in the following results.

\section{Results}

Table 1 shows the percentage of respondents who say the statements about evolution, the Big Bang, and continental drift are false, as well as those saying the sun revolves around the earth. Fifty percent disbelief in evolution is consistent with earlier results. Disbelief in the Big Bang, is slightly higher, though the difference is insignificant. In contrast, few Americans doubt continental drift. Twice as many $(20 \%)$ erroneously say the sun revolves around the earth. There is high overlap between those who disbelieve evolution and those doubting the Big Bang $(r=0.64, p<0.001)$.
Table 1 Percentage of Americans not accepting basic concepts of Earth science

\begin{tabular}{lllll}
\hline Basic concept: & Human evolution & Big Bang & Drifting continents & Heliocentric universe \\
\hline Percent disbelieving: & $50 \%$ & $52 \%$ & $10 \%$ & $20 \%$ \\
\hline
\end{tabular}


Table 2 Predictors (Betas) of incorrect beliefs about Earth science

$* p<0.01$

$* * p<0.001$

\begin{tabular}{lllll}
\hline & Human evolution & Big Bang & Drifting continents & Heliocentric universe \\
\hline Fundamentalist religiosity & $0.32^{* *}$ & $0.30^{* *}$ & $0.13^{* *}$ & 0.02 \\
Education & $-0.15^{* *}$ & $-0.13^{* *}$ & $-0.06^{*}$ & $-0.19^{*}$ \\
Political conservatism & $0.18^{* *}$ & $0.16^{* *}$ & $0.08^{*}$ & 0.04 \\
$R^{2}$ & 0.45 & 0.41 & 0.19 & 0.21 \\
\hline
\end{tabular}

Pertinent independent variables-religiosity, education, and political conservatism - are used in an OLS regression model to predict each incorrect belief. The effects of each independent variable (along with significance levels) are shown in Table 2 as betas. We see that fundamentalist religiosity, with beta $=0.32$, is the independent variable most strongly related to disbelief in human evolution. Education (beta $=-0.15$ ) is a weaker and negative predictor, meaning that people with little education are somewhat more likely than those well-educated to disbelieve in evolution. Political conservatives are more likely than liberals to disbelieve in evolution.

Predictors (betas) of doubting the Big Bang are virtually the same as those for disbelief in evolution. Fundamentalist religiosity has by far the strongest effect in both cases. Low education and political conservatism contribute lesser but about equal amounts, independently of religiosity.

Religiosity and low education only slightly influence disbelief in continental drift. Only low education predicts confusion about whether the sun or earth is the center of rotation.

The persistent covariation of political view with "Big Bang" beliefs, despite statistical controls, is illustrated in Fig. 1, which is limited to subjects who are not in fundamentalist religious denominations. Here, the percentage of respondents believing that the universe began with a huge explosion is plotted simultaneously against political view (conservative, moderate, and liberal) and education (high school or less, some college, and graduate school). As a cautionary note, subsample sizes are small, ranging from 26 to 97 . However, the consistency of results - across all educational groups-is striking. Among nonfundamentalist respondents, whatever their education level, belief in the Big Bang rises along with political liberalism.

\section{Discussion}

Half of Americans do not believe in the Big Bang, virtually the same percentage that rejects human evolution. Fundamentalist religiosity is twice as strong a predictor of both false beliefs as either low education or political conservatism. The virtually identical regression models for disbelief in the Big Bang and disbelief in evolution suggest that both rejections of modern earth science are drawn from the same wellspring.
The strong association between fundamentalist religiosity and disbelief in evolution is not surprising. The Bible's two creation myths, one about seven days (Genesis 1), the other about Adam and Eve (Genesis 2), explicitly contradict Darwinian evolution. Indeed, they contradict one another. In the seven days story, all vegetation including seed plants and fruit trees is made on the third day. All sea creatures and flying birds are made on the fifth day. All land animals from cattle to creeping things are made on the sixth day, and afterward God makes humans - male and female - to rule over these fish, birds, and animals, and to use the plants for food.

In the second story, Adam comes first "when no plant of the field was yet on the earth." Then plants are created in Eden. Then "out of the ground Yahweh formed every animal of the field and every bird of the air." Finally Eve is made from Adam's rib. (See Genesis 2: 6-22, New Revised Standard Version Bible 1989.)

Was the first man created before plants and animals and birds, or afterward? Did birds appear before land animals or at the same time? The sequences agree on only two points: (1) vegetation preceded animals and birds, and (2) the first woman was created at the end of the process. There is little correspondence between either of the biblical sequences and our modern understanding of life's history (Fortey 1999).

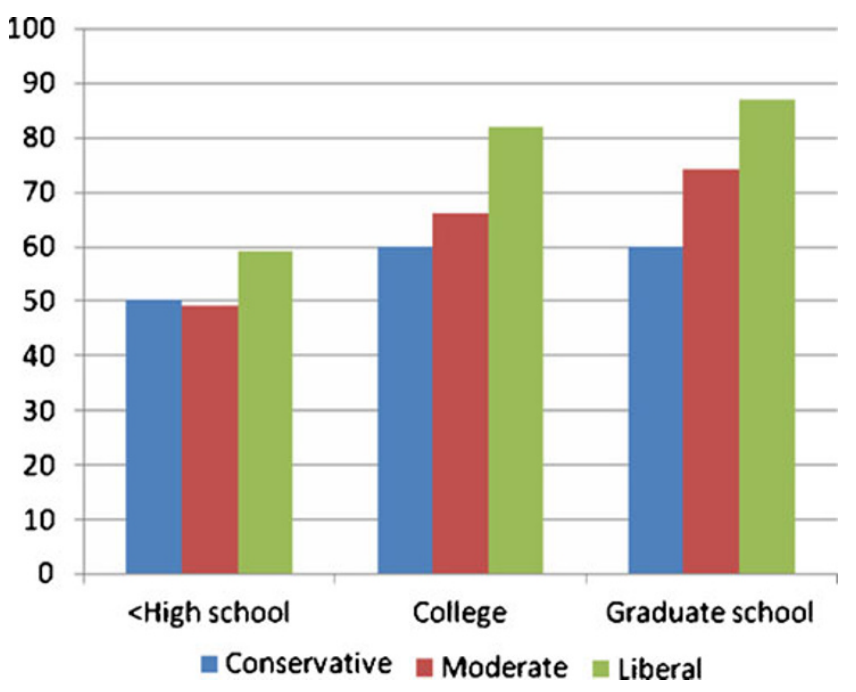

Fig. 1 Non-fundamentalists Only: Percent Believing in the Big Bang, by Education and Political View 
Trilobites are among the earliest fossils of complex animals, appearing long before land plants. Plant and animal life was abundant on land before many kinds of fish appeared; and marine mammals including whales are relatively recent. Land plants did precede land animals that fed on them, but seed plants and fruit trees (angiosperms) appeared after dinosaurs and small mammals had long roamed the earth. Birds followed (probably descended from) dinosaurs. Humans - of both sexes - are the newest of the major kinds mentioned in Genesis. The remarkably high persistence of belief in the literal word of the Bible, despite its internal contradictions and contrary scientific evidence, is an enigma of modern American culture (Mazur 2007b).

There is an equally strong association of religious fundamentalism with disbelief in the Big Bang. Conceivably, the biblical creation of light on the "first day" might encourage a belief in the sudden appearance of the universe. That interpretive possibility notwithstanding, the idea of a big bang is as unappealing to biblical literalists as Darwinism.

In contrast, it is remarkable that only ten percent of Americans doubt the notion of drifting continents, an idea that until the 1960s was generally rejected by earth scientists. Religious fundamentalists are only slightly charier of the idea than non-fundamentalists, even though the question explicitly refers to continental movement over "millions of years," a wording that ought to be unacceptable to anyone believing the earth is only 6,000 years old.

Why do Americans, many of whom reject evolution and the Big Bang, so readily accept continental drift? Of course the scientific evidence accumulated in the 1960s and afterward makes a compelling case for spreading ocean floors and has caused a revolution in the earth sciences (Oreskes 2003), but it is implausible that a large portion of the public would know or understand these findings. The general idea of moving continents is widely known in American culture, and since it does not contradict scripture, there is apparently little reason to doubt it.

I was surprised to find more acceptance of continental drift than of the heliocentric solar system. Some erroneous answers to the sun-earth question may be partly due to confusion about question wording. On the other hand, the association between wrong answers about the sun-centered universe and low education suggests that there is real misunderstanding on so fundamental a fact. Still, overall, poor education is not as high a barrier as fundamentalist religiosity to public understanding of earth science.

The most peculiar finding here is that political conservatism is associated with disbelief in the Big Bang and in evolution even among non-fundamentalist respondents who are well-educated (Fig. 1). This reliable result defies commonsense interpretation.

Why should well-educated political conservatives, who are not committed to a literal Bible, be especially prone to reject central principles of modern earth science? The answer, I think, lies first in realizing that most educated people do not thoroughly understand the biological, geological, and astronomical evidence that makes scientifically unassailable cases for the evolution of life and the expansion of the universe. Most nonscientists accept (or reject) these concepts largely on the word of authorities whom they respect or on views that are current in their circles (Ruse 2005). Furthermore, in American politics during the last few decades, there has been increasing left-right polarization on a number of "hot button" issues including abortion, the Iraq war, immigration, climate change, and teaching "intelligent design" in public schools. Citizens leaning toward each extreme tend to adopt the issues of their fellow extremists (Hetherington and Weiler 2009). Conservatives with no particular interest in the Bible tend to accept the positions vigorously espoused by their coalition mates on the religious right. There is no reason to doubt that this is a symmetrical effect, working as well on liberals.

In principle, a biblical literalist could object to plate tectonics with its implication of an earth that is millions if not billions of years old. But in practice, continental drift has not been drawn into the political fray. How might an educator use this information? Earth science teachers, facing students wary about Darwinism, might begin their courses by explaining continental drift since nearly everyone believes it is true. This opens a smooth path for instruction about the ancient earth, the millennia-long processes of deposition of sediments and erosion, the comparison of fossils found in lower versus higher rock strata, and how these changes in these fossils reveal endless forms most beautiful and most wonderful.

\section{References}

Agresti A. Categorical Data Analysis. New York: Wiley-Interscience; 2002. Fortey R. Life: a Natural History of the First Four Billion Years of Life. New York: Vintage; 1999.

Hetherington M, Weiler J. Authoritarianism and Polarization in American Politics. New York: Cambridge University Press; 2009.

Mazur A. "Believers and Disbelievers in Evolution". Polit Life Sc. 2004;23 (September):55-61.

Mazur, A. "Disbelievers in Evolution." Science. 2007a;315 (January 12): 184

Mazur A. Implausible Beliefs in the Bible, Astrology, and UFOs. New Brunswick, NJ: Transaction Publications; 2007b.

Miller J, Scott E, S. Okamoto. "Public Acceptance of Science." Science. 2006;313 (August 11):765-766.

New Revised Standard Version Bible. New York: Oxford University Press; 1989.

Oreskes N. Plate Tectonics: An Insider's History of the Modern Theory of the Earth. Boulder CO: Westview Press; 2003.

Ruse M. The Evolution-Creation Struggle. Cambridge MA: Harvard University Press; 2005.

Smith T. Classifying Protestant Denominations. Chicago: National Opinion Research Corporation; 1987. 